\title{
Left Main Protection and Emergency Stenting During TAVR with Self-Expandable Valve
}

\author{
Marko Noc, MD, PhD ${ }^{1,2^{*}}$, Branko Cveticanin, MD ${ }^{1,3}$, Saibal Kar, MD ${ }^{1,4}$, Oscar A. Mendiz, MD ${ }^{1,5}$ \\ ${ }^{1}$ MC Medicor, Izola, Slovenia \\ ${ }^{2}$ Center for Intensive Internal Medicine, University Medical Center, Ljubljana, Slovenia \\ ${ }^{3}$ Department of Radiology, General Hospital Izola, Izola, Slovenia \\ ${ }^{4}$ Cardiovascular Intervention Center, Cedars-Sinai Heart Institute, Los Angeles, California, United States \\ ${ }^{5}$ Interventional Cardiology Department, Cardiology \& Cardiovascular Institute. Hospital Universitario Fundación Favaloro, \\ Buenos Aires, Argentina
}

\begin{abstract}
Left main (LM) obstruction is rare but life-threatening complication of transcatheter aortic valve replacement (TAVR) which occurs by displacement of left coronary leaflet toward the ostium or by direct occlusion by the covered skirt of the prosthesis. We report an 88year old lady with severe aortic stenosis, short distance from annulus to left main origin, shallow/low sinus of Valsalva, and calcification of the left aortic leaflet undergoing TAVR with a self-expandable valve. Instead of recently described "Chimney" stenting with protrusion of a very long stent segment from LM above the prosthesis leaflets and behind the valve frame, a "T-stenting" with stent protrusion only into the left sinus Valsalva was used to secure the LM patency.

Copyright $\odot 2018$ Science International Corp.
\end{abstract}

\section{Key Words \\ Emergency left main stenting, TAVR \\ Introduction}

Left main (LM) obstruction is rare but life-threatening complication of transcatheter aortic valve replacement (TAVR) which occurs by displacement of left coronary leaflet over the ostium or by direct occlusion by the covered skirt of the prosthesis [1, 2].

Fax +1 2037853346

E-Mail: jshd@scienceinternational.org

http://structuralheartdisease.org/

\author{
(C) 2018 Journal of Structural Heart Disease \\ Published by Science International Corp. \\ ISSN 2326-4004 \\ Accessible online at: \\ http://structuralheartdisease.org/
}

Since this complication may be anticipated if a careful evaluation of aortic computed tomography (CT) scan is performed, LM can be protected up front by placement of a guiding catheter into the LM ostium and advancing a guidewire with undeployed stent into the left anterior descending artery (LAD) [2]. If LM occlusion occurs, the stent can be immediately withdrawn and deployed to reestablish LM patency. Importantly, because of the valve height during the TAVR with a self-expandable valve such as Evolut $R$ (Core Valve Evolut R, Medtronic, Dublin, Ireland), the guiding catheter is located behind rather than above the valve frame. In case of LM occlusion, a "chimney" stenting with protrusion of a very long stent segment from the LM ostium above the prosthesis leaflets and behind the valve frame, has recently been described [3]. We herein present an alternative "T-stenting" strategy with stent protrusion extending only into the sinus of Valsalva toward the valve frame without leaving any stent segment behind the valve frame.

\section{Case report}

The patient was an 88-year-old lady with symptomatic severe aortic stenosis (gradient $69 / 37 \mathrm{~mm}$ $\mathrm{Hg}$, AVA $0.4 \mathrm{~cm} 2$ ) and preserved left ventricular ejec-

\footnotetext{
* Corresponding Author:

Marko Noc, MD, PhD

Center for Intensive Internal Medicine

University Medical Center

Zaloska 7, 1000 Ljubljana, Slovenia

Tel. +386 41723 807; Fax: +386 152222 90; E-Mail: marko.noc@mf.uni-lj.si
} 

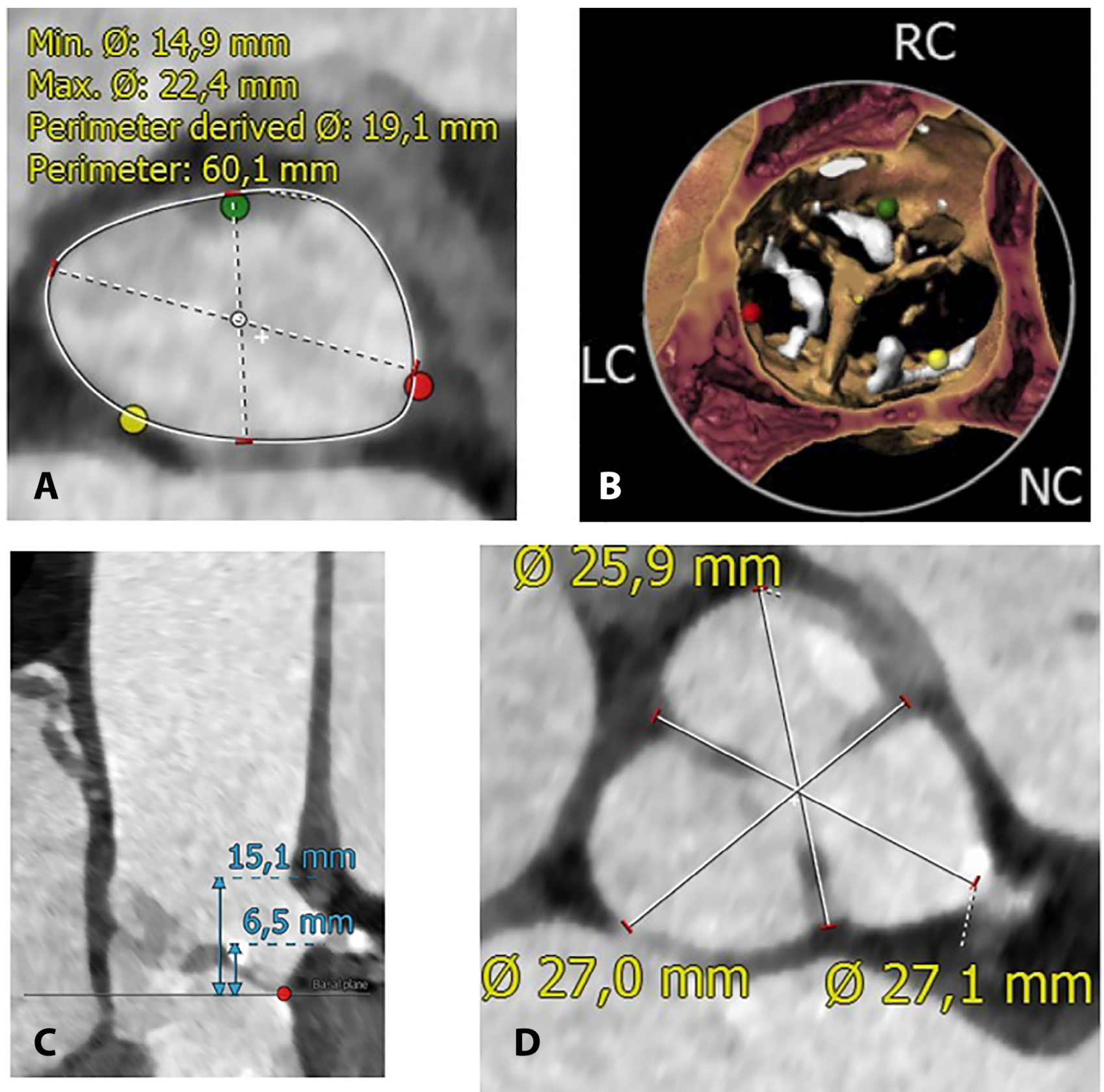

Figure 1. CT measurements of aortic annulus (Panel A), aortic leaflet calcification (Panel B), height of left coronary ostia/left sinus of Valsalva (Panel C) and diameters of sinuses of Valsalva (Panel D).

tion fraction (72\%) who was referred for TAVR by the Heart Team because of very high surgical risk (Euroscore II $15.30 \%$, STS $11.06 \%)$. Coronary angiography showed diffuse calcification and moderate proximal and mid LAD disease. Pre-procedural CT scan revealed aortic annulus perimeter of $60.1 \mathrm{~mm}$, short distance from annulus to LM origin $(6.5 \mathrm{~mm})$, shallow sinuses of Valsalva (average $26.7 \mathrm{~mm}$ ), a borderline height of left sinus Valsalva $(15.1 \mathrm{~mm})$ and a calcified nodule on the left coronary cusp (Figure 1). Because of threatened LM occlusion during valve placement, an EBU 3.56 Fr guiding catheter (Medtronic, Dublin, Ireland) was placed via left radial artery to cannulate LM. A .014" BMW guidewire (Abbott Vascular, Abbott 


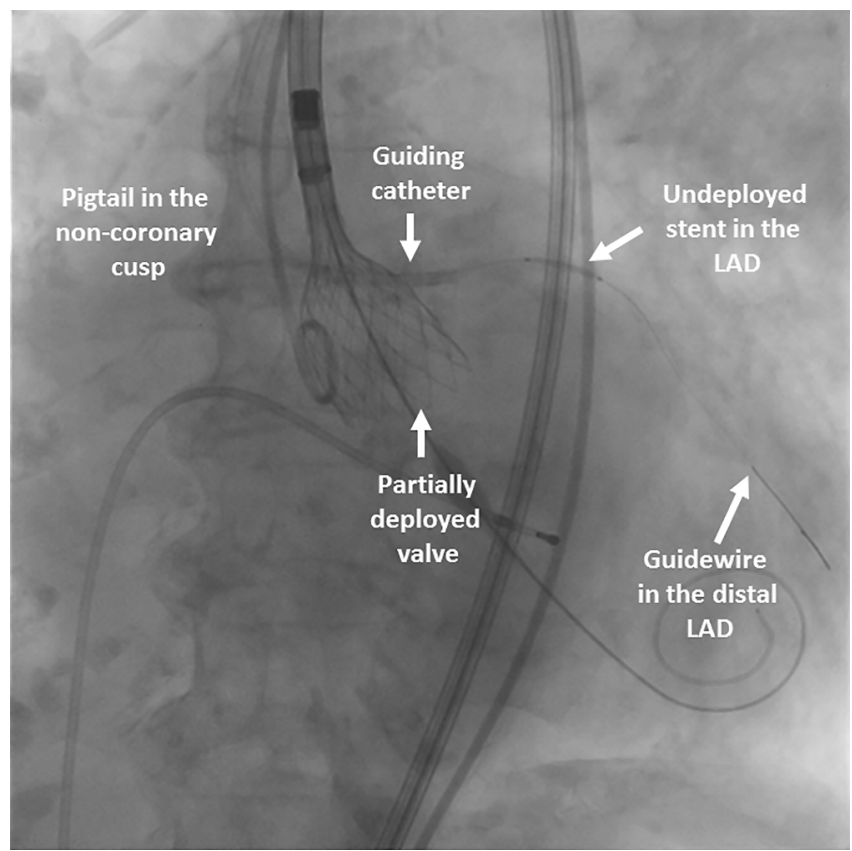

Figure 2. Valve deployment to the point of no recapture with the guiding catheter lying behind the valve frame, and guidewire with undeployed stent in the left anterior descending artery.

Park, Illinois, USA) was advanced into LAD followed by placement of undeployed drug-eluting stent Orsiro 3.5x15 (Biotronik, Berlin, Germany). Using the right femoral artery, a $26 \mathrm{~mm}$ self-expandable Evolut $\mathrm{R}$ valve (Core Valve Evolut R, Medtronic, Dublin, Ireland) was deployed without predilation (Figure 2). Left coronary flow, assessed prior to full deployment, was preserved (Video 1). However, after full deployment, aortography revealed decreased left coronary compared to right coronary flow despite pulling the stent back to the guiding catheter while still maintaining guidewire position (Video 2). Guide injection showed that left leaflet was displaced close to the LM ostium (Video 3). The stent was re-advanced to the LM, protruded proximally close to the valve frame and deployed (Video 4). After placement of an LM stent which extended into the proximal LAD, a haziness was noticed at the distal edge. Stented segment was initially extended with a $2.5 \times 12$ Orsiro (Biotron$i k$, Berlin, Germany). Since haziness persisted, additional $2.5 \times 15 \mathrm{~mm}$ Orsiro was deployed distally and this resulted in a good angiographic result (Video 5). Aortogram revealed widely patent LM, LAD and circumflex artery with normal flow (Video 6). Except for

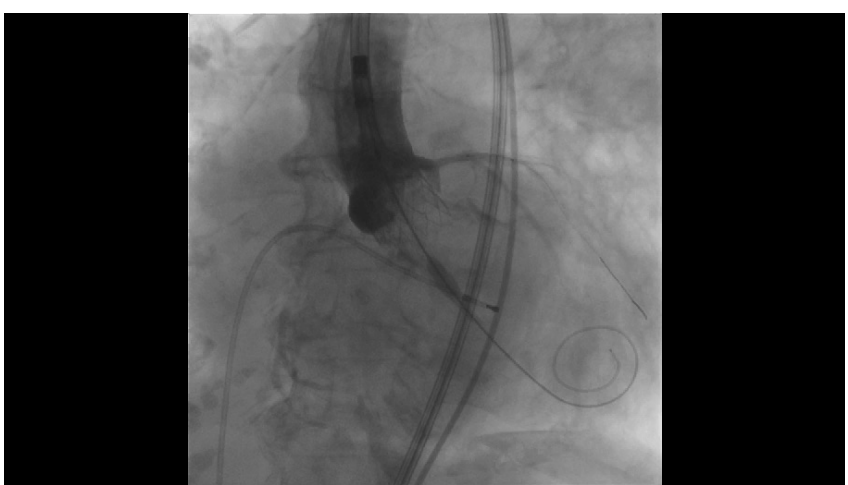

Video 1. Aortogram before complete valve deployment. Both coronary arteries are well perfused. View supplemental video at https://doi.org/10.12945/j.jshd.2018.008.18.vid.01.

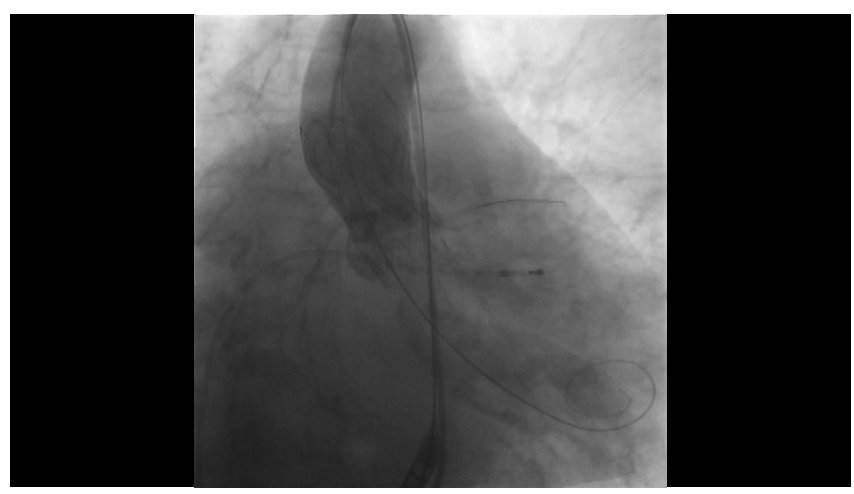

Video 2. Aortogram with decreased left compared to right coronary flow after complete valve deployment despite moving of the stent from LAD back to the guiding catheter. View supplemental video at https://doi.org/10.12945/j.jshd.2018.008.18. vid.02.

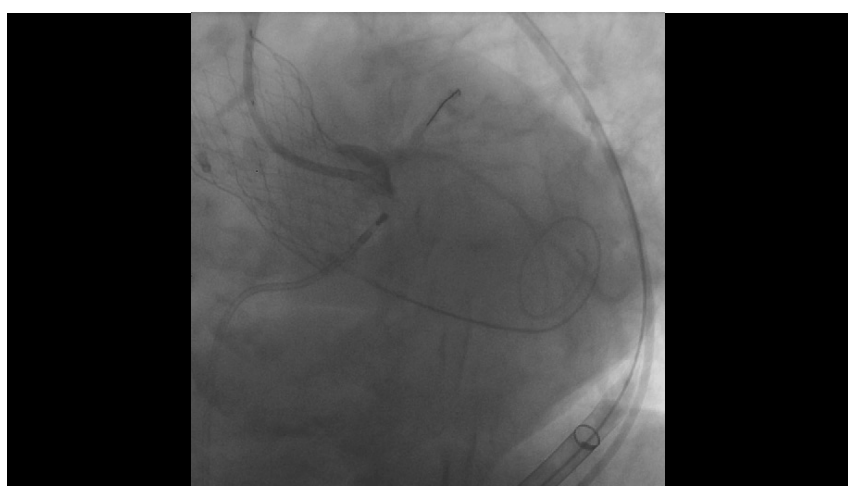

Video 3. Injection through the guiding catheter revealed a mass protruding toward the LM ostium. View supplemental video at https://doi.org/10.12945/j.jshd.2018.008.18.vid.03.

the need for a permanent pacemaker, the hospital stay was uneventful and the patient was discharged with dual antiplatelet therapy including acetylsalicyl- 


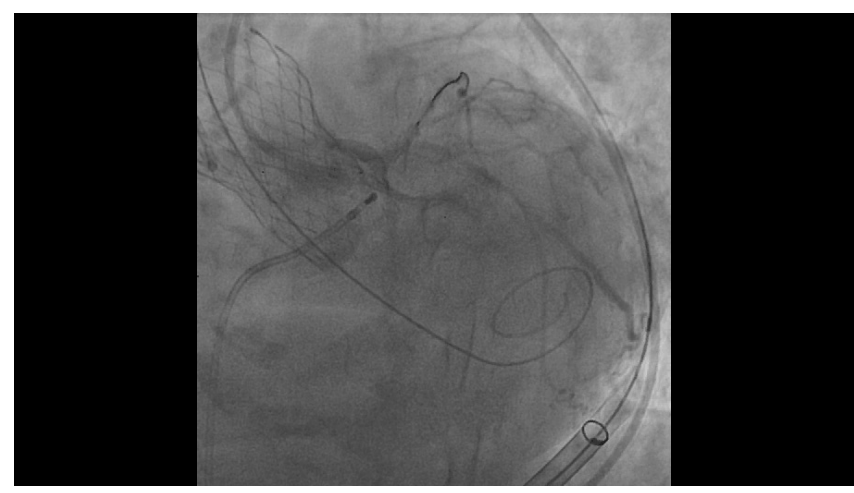

Video 4. Stent deployment from the LM toward the valve frame. View supplemental video at https://doi.org/10.12945/j. jshd.2018.008.18.vid.04.

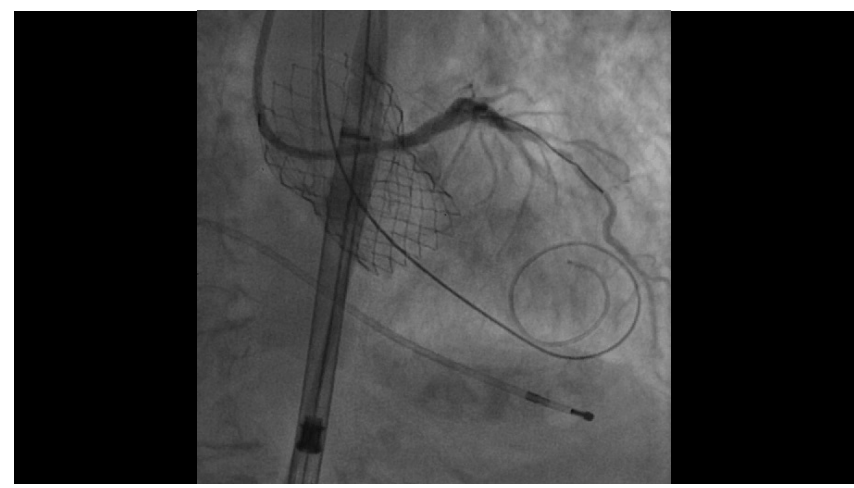

Video 5. Post procedural guide injection showed widely patent LM, LAD and left circumflex artery with normal flow. View supplemental video at https://doi.org/10.12945/j.jshd.2018.008.18. vid.05

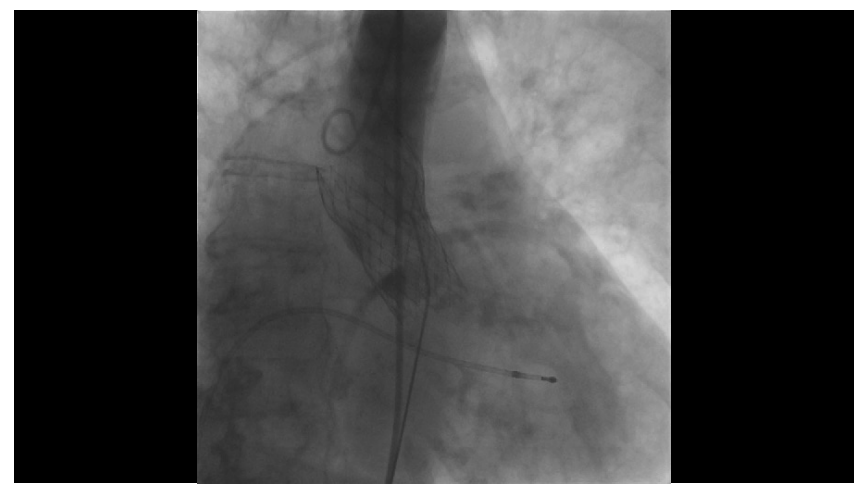

Video 6. Final aortogram with comparable left and right coronary flow. View supplemental video at https://doi.org/10.12945/j. jshd.2018.008.18.vid.06.

ic acid and clopidogrel. Post-procedural CT scan after three weeks revealed widely patent LM stent protruding across the sinus of Valsalva close to valve frame with its proximal part in a "T-stent" shape. (Figure 3 ). Twelve months after the intervention, the patient continues to be asymptomatic.

\section{Discussion}

Several anatomic factors derived from preprocedural CT scan including low LM ostium, shallow sinuses of Valsalva, severe leaflet calcification with large bulky calcium nodules, high native leaflet length/ curved coronary sinus height ratio as well as extreme valve oversizing and "valve-in-valve" procedure, have been identified as high risk features for coronary occlusion during TAVR $[2,4]$. A potential risk of coronary occlusion may also be assessed before TAVR using aortic valve predilatation with simultaneous aortogram. If the coronary occlusion is documented, upfront LM protection is mandatory [4]. We did not use this technique because we perform a vast majority of TAVR without predilatation. If we predilate, we always use a small balloon (18-20 $\mathrm{mm}$ ) to minimize manipulation of the calcified native valve. Predilatation with a smaller balloon would probably underestimate the likelihood of actual LM occlusion during TAVR. Moreover, based on the CT scan, we have already decided to use upfront LM protection. Admittedly, according to CT-derived measurements, a $23 \mathrm{~mm}$ rather than 26 $\mathrm{mm}$ Evolut $\mathrm{R}$ should have been used. Some oversizing with this self-expandable prosthesis was selected because annulus perimeter was at the very upper limit for the $23 \mathrm{~mm}$ valve, the native valve was highly calcified, and we have already decided for upfront LM protection.

The optimal strategy for LM protection during TAVR, particularly when using self-expandable Evolut $R$ valve, remains to be defined. The "chimney" technique is generally considered in patients with low sinotubular junction due to potential occlusion of the respective sinus of Valsalva after valve deployment. In our patient, the height of the left sinus of Valsalva was just above the recommended $15 \mathrm{~mm}$. We, therefore, decided for a less complex "T-stenting" and protrude LM stent only above the displaced leaflet and toward the valve frame. Accordingly, the proximal part of the stent was not behind the valve frame. This avoids possible unfavorable interaction between the LM stent and self-expanding valve frame which 


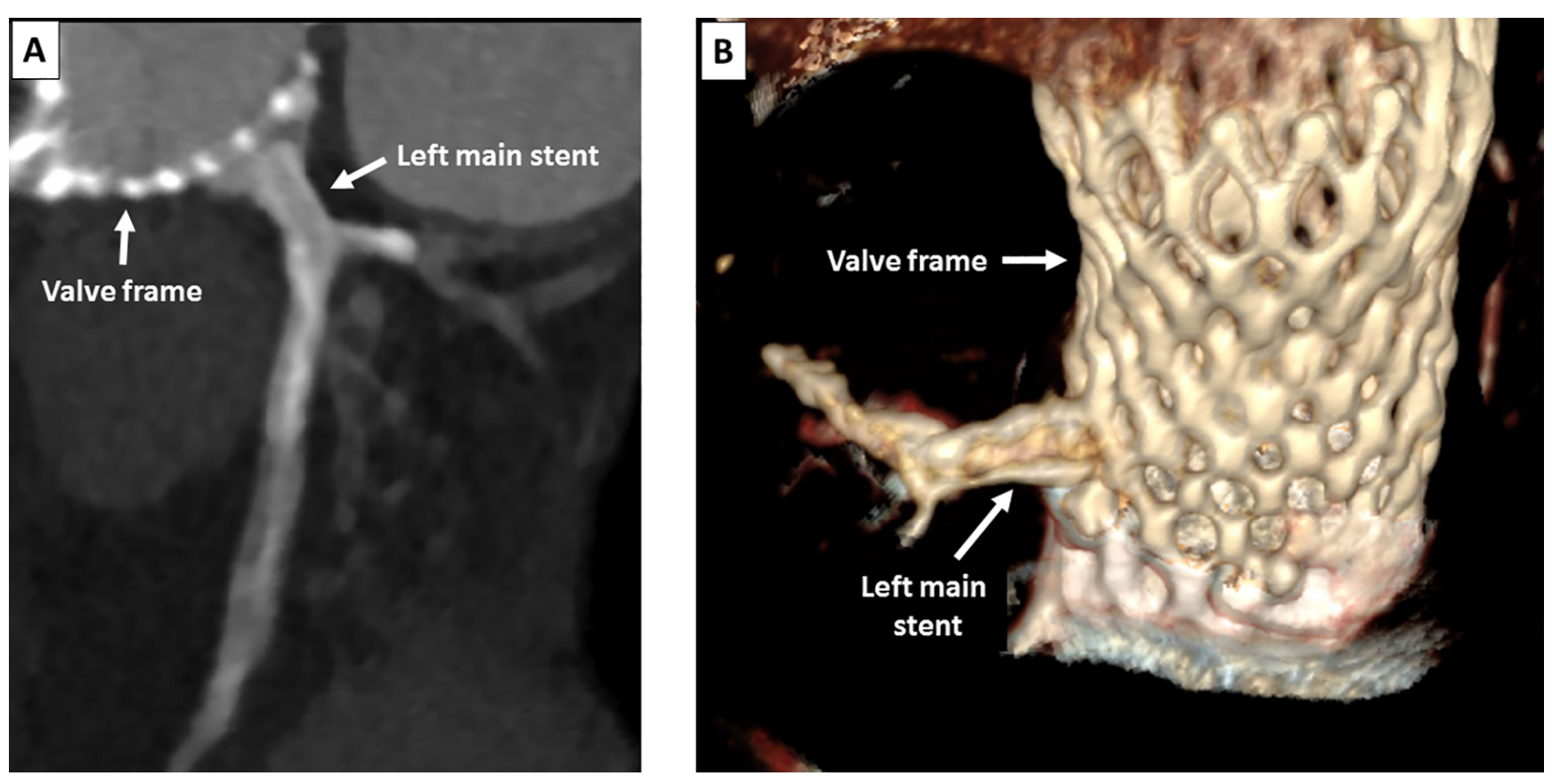

Figure 3. Post procedural computed tomography scan after 3 weeks showing widely patent LM stent protruding toward the valve frame (Panel $A$ ) together with three-dimensional reconstruction (Panel B).

may also happen after the procedure. We also believe that smaller stent protrusion may facilitate left coronary engagement in the future and may theoretically reduce the risk of stent thrombosis. Of note, using our "T-stenting" technique, we were able to deliver an additional two stents to the LAD through the LM stent without any problems. Retrospectively, we believe that additional LAD stenting was required because there was a distal edge dissection from the initial LM stent which extended into the diffusely diseased proximal and mid LAD. Beside "chimney" and our "T-stenting" techniques, the third option to prevent LM occlusion during TAVR is the recently described "BASILICA" technique with modulating of the native valve leaflet. A single leaflet tear, which would prevent coronary occlusion, is made by leaflet wire traversal and snaring followed by slicing. To our knowledge, this technique has been described so far in only six "valve-in-valve" procedures and one native aortic stenosis. We did not use "BASILICA" because of the lack of experience and fear to increase stroke risk when manipulating with a heavily calcified cusp. However, when discussing any of the herein described LM protection techniques during TAVR, it is important to no- tice that the number of reported patients is still very limited and long-term efficacy and safety remains to be proven.

\section{Acknowledgements}

We are grateful to Natasa Suligoj Cernic, MD for echocardiography and MC Medicor chief medical officer, Metka Zorc, MD, PhD.

\section{Conflict of Interest}

Marko Noc and Branko Cveticanin have no relevant conflict of interest.

Saibal Kar reports research grant from Edwards Lifesciences and research grants and consultation fees from Abbott Vascular and Boston Scientific

Oscar Mendiz reports proctorship for CoreValve (Medtronic) and consultation fees from Abbott Vascular.

Comment on this Article or Ask a Question 


\section{References}

1. Ribeiro $H B$, Nombela-Franco $L$, Urena $M$, Mok M, Pasian S, Doyle D, et al. Coronary obstruction following transcatheter aortic valve implantation: a systematic review. JACC Cardiovasc Interv. 2013;6:452-461. DOI: 10.1016/j.jcin.2012.11.014

2. Abramowitz $Y$, Chakravarty $T$, Jilaihawi $H$, Kashif M, Kazuno Y, Takahashi N, et al. Clinical impact of coronary protection during transcatheter aortic valve implantation: first reported series of patients. Eurointervention. 2015;11:572-581. DOI: 10.4244/ EIJV11I5A112
3. Spaziano M, Akodad M, Hovasse T, Lefèvre $T$, Bouvier E, Chevalier B. Simultaneous TAVR and left main "Chimney" stenting in a patient with low left main height. JACC Cardiovasc Interv. 2017;10:e185-e187. DOI: 10.1016/j.jcin.2017.06.047

4. Dvir D, Leipsic J, Blanke P, Ribero HB, Kornowski R, Pichard A, et al. Coronary obstruction in transcatheter aortic valvein-valve implantation: preprocedural evaluation, device selection, protection and treatment. Circ Cardiovasc Interv. 2015 Jan;8(1). pii:e002079. DOI: 10.1161/CIRCINTERVENTIONS.114.002079
Cite this article as: Noc $M$, Cveticanin B, Kar S, Mendiz OA. Left Main Protection and Emergency Stenting During TAVR with Self-Expandable Valve. Structural Heart Disease. 2018;4(5):240245. DOI: https://doi.org/10.12945/j. jshd.2018.008.18 